\title{
The saturation of several universal inequalities in information-processing
}

\author{
Lin Zhang ${ }^{1, *}$, Junde $\mathrm{Wu}^{2,+}$, Shao-Ming $\mathrm{Fei}^{3}$ \\ ${ }^{1}$ Institute of Mathematics, Hangzhou Dianzi University, Hangzhou 310018, PR China \\ ${ }^{2}$ Department of Mathematics, Zhejiang University, Hangzhou 310027, PR China \\ ${ }^{3}$ School of Mathematics of Sciences, Capital Normal University, Beijing 100048, PR China
}

\begin{abstract}
In this paper, we characterize the saturation of four universal inequalities in quantum information theory, including a variant version of strong subadditivity inequality for von Neumann entropy, the coherent information inequality, the Holevo quantity and average entropy inequalities. These results shed new light on quantum information inequalities.
\end{abstract}

Keywords: Strong subadditivity; Coherent information; Holevo quantity; Quantum channel

\section{Introduction and preliminaries}

Let $\mathcal{H}$ be a finite dimensional complex Hilbert space. A quantum state $\rho$ on $\mathcal{H}$ is a positive semidefinite operator of trace one, in particular, for each unit vector $|\psi\rangle \in \mathcal{H}$, the operator $\rho=|\psi\rangle\langle\psi|$ is said to be a pure state. The set of all quantum states on $\mathcal{H}$ is denoted by $\mathrm{D}(\mathcal{H})$. For each quantum state $\rho \in \mathrm{D}(\mathcal{H})$, its von Neumann entropy is defined by $\mathrm{S}(\rho)=-\operatorname{Tr}\left(\rho \log _{2} \rho\right)$.

In quantum information theory, the strong subadditivity inequality of von Neumann entropy, proved by Lie and Ruskai in [1], stated that

$$
\mathrm{S}\left(\rho_{A B C}\right)+\mathrm{S}\left(\rho_{B}\right) \leqslant \mathrm{S}\left(\rho_{A B}\right)+\mathrm{S}\left(\rho_{B C}\right) .
$$

*E-mail: godyalin@163.com; linyz@zju.edu.cn

${ }^{\dagger}$ Corresponding author. E-mail: wjd@zju.edu.cn 
The strong subadditivity inequality of von Neumann entropy is ubiquitous, for example, as some direct consequences, the data processing inequality, the well-known Holevo bound [2], in particular, it connects with the monotonicity of relative entropy under quantum channels [3].

If a reference system $D$ is introduced such that $\rho_{A B C D}$ is the purification of $\rho_{A B C}$, that is, $\rho_{A B C D}$ is a pure state with $\rho_{A B C}=\operatorname{Tr}_{D}\left(\rho_{A B C D}\right)$, then an equivalent version of strong subadditivity inequality of von Neumann entropy can be described by [4]:

$$
\mathrm{S}\left(\rho_{D}\right)+\mathrm{S}\left(\rho_{B}\right) \leqslant \mathrm{S}\left(\rho_{A B}\right)+\mathrm{S}\left(\rho_{A D}\right) .
$$

A quantum channel $\Phi$ on $\mathcal{H}$ is a trace-preserving completely positive linear mapping defined on the set $\mathrm{D}(\mathcal{H})$. It follows from ([5, Prop. 5.2 and Cor. 5.5]) that there exists linear operators $\left\{K_{\mu}\right\}_{\mu}$ on $\mathcal{H}$ such that $\sum_{\mu} K_{\mu}^{\dagger} K_{\mu}=\mathbb{1}$ and for each quantum state $\rho$, we have the Kraus representation

$$
\Phi(\rho)=\sum_{\mu} K_{\mu} \rho K_{\mu}^{\dagger}
$$

The complementary channel $\widehat{\Phi}$ of $\Phi$ acts on quantum state $\rho$ is defined by [2]:

$$
\widehat{\Phi}(\rho)=\sum_{\mu, v} \operatorname{Tr}\left(K_{\mu} \rho K_{v}^{+}\right)|\mu\rangle\langle v|,
$$

the von Neumann entropy $S(\widehat{\Phi}(\rho))$ of $\widehat{\Phi}(\rho)$ is said to be the exchange entropy. For the topics related to complementary channel, the readers can referred to [6].

The Coherent information $I_{\mathcal{c}}(\rho, \Phi)$ can be defined by the difference of von Neumann entropy S $(\Phi(\rho))$ of output quantum state $\Phi(\rho)$ and the exchange entropy [7]:

$$
I_{c}(\rho, \Phi)=\mathrm{S}(\Phi(\rho))-\mathrm{S}(\widehat{\Phi}(\rho))
$$

It follows from the strong subadditivity inequality of von Neumann entropy that the coherent information $I_{c}(\rho, \Phi)$ can be bounded by the entropy $S(\rho)$ of the initial state $S(\rho)$, that is

$$
I_{c}(\rho, \Phi) \leqslant S(\rho)
$$

Let $\mathcal{E}=\left\{\left(p_{\mu}, \rho_{\mu}\right)\right\}$ be a quantum ensemble on $\mathcal{H}$, that is, each $\rho_{\mu} \in \mathrm{D}(\mathcal{H}), p_{\mu}>0$, and $\sum_{\mu} p_{\mu}=1$. The Holevo quantity of the quantum ensemble $\left\{\left(p_{\mu}, \rho_{\mu}\right)\right\}$ is defined by

$$
\chi\left\{\left(p_{\mu}, \rho_{\mu}\right)\right\}=\mathrm{S}\left(\sum_{\mu} p_{\mu} \rho_{\mu}\right)-\sum_{\mu} p_{\mu} \mathrm{S}\left(\rho_{\mu}\right) .
$$

Let $\rho$ be a quantum state, and $\Phi(*)=\sum_{\mu} K_{\mu}(*) K_{\mu}^{\dagger}$ be a quantum channel. If we denote

$$
q_{\mu}=\operatorname{Tr}\left(K_{\mu} \rho K_{\mu}^{+}\right), \quad \rho_{\mu}^{\prime}=q_{\mu}^{-1} K_{\mu} \rho K_{\mu}^{+}
$$


and

$$
\rho^{\prime}=\sum_{\mu} q_{\mu} \rho_{\mu}^{\prime}
$$

then $\Phi$ induced a quantum ensemble $\left\{q_{\mu}, \rho_{\mu}^{\prime}\right\}$.

In [2], Roga proved that the Holevo quantity $\chi\left(\left\{q_{\mu}, \rho_{\mu}^{\prime}\right\}\right)$ of quantum ensemble $\left\{q_{\mu}, \rho_{\mu}^{\prime}\right\}$ can be bounded by the exchange entropy $S(\widehat{\Phi}(\rho))$, and the average entropy $\sum_{\mu} q_{\mu} S\left(\rho_{\mu}^{\prime}\right)$ of $\left\{q_{\mu}, \rho_{\mu}^{\prime}\right\}$ can be bounded by the entropy $S(\rho)$ of the initial state $\rho$, that is

$$
\chi\left(\left\{q_{\mu}, \rho_{\mu}^{\prime}\right\}\right) \leqslant S(\widehat{\Phi}(\rho))
$$

and

$$
\sum_{\mu} q_{\mu} \mathrm{S}\left(\rho_{\mu}^{\prime}\right) \leqslant \mathrm{S}(\rho)
$$

In [8], the saturation of strong subadditivity inequality $S\left(\rho_{A B C}\right)+\mathrm{S}\left(\rho_{B}\right) \leqslant \mathrm{S}\left(\rho_{A B}\right)+\mathrm{S}\left(\rho_{B C}\right)$ of von Neumann entropy is presented:

Proposition 1.1 ([8]). A state $\rho_{A B C} \in \mathrm{D}\left(\mathcal{H}_{A} \otimes \mathcal{H}_{B} \otimes \mathcal{H}_{C}\right)$ saturates the strong subadditivity inequality of von Neumann entropy, that is,

$$
\mathrm{S}\left(\rho_{A B}\right)+\mathrm{S}\left(\rho_{B C}\right)=\mathrm{S}\left(\rho_{A B C}\right)+\mathrm{S}\left(\rho_{B}\right)
$$

if and only if there is a decomposition of system $B$ as

$$
\mathcal{H}_{B}=\bigoplus_{j} \mathcal{H}_{b_{j}^{L}} \otimes \mathcal{H}_{b_{j}^{R}}
$$

such that

$$
\rho_{A B C}=\bigoplus_{j} \lambda_{j} \rho_{A b_{j}^{L}} \otimes \rho_{b_{j}^{R} C^{\prime}}
$$

where $\rho_{A b_{j}^{L}} \in \mathrm{D}\left(\mathcal{H}_{A} \otimes \mathcal{H}_{b_{j}^{L}}\right), \rho_{b_{j}^{R} C} \in \mathrm{D}\left(\mathcal{H}_{b_{j}^{R}} \otimes \mathcal{H}_{C}\right),\left\{\lambda_{j}\right\}$ is a probability distribution.

Let $\rho_{B C} \in \mathrm{D}\left(\mathcal{H}_{B} \otimes \mathcal{H}_{C}\right), \rho_{B}=\operatorname{Tr}_{C}\left(\rho_{B C}\right), \rho_{C}=\operatorname{Tr}_{B}\left(\rho_{B C}\right)$. The famous Araki-Lieb inequality showed that

$$
\left|S\left(\rho_{B}\right)-S\left(\rho_{C}\right)\right| \leqslant S\left(\rho_{B C}\right)
$$

In [9], the saturation of Araki-Lieb inequality is presented:

Proposition $1.2([9]) . S\left(\rho_{B C}\right)=S\left(\rho_{B}\right)-S\left(\rho_{C}\right)$ if and only if 
(i) $\mathcal{H}_{B}$ can be decomposed into $\mathcal{H}_{B}=\mathcal{H}_{L} \otimes \mathcal{H}_{R}$,

(ii) $\rho_{B C}=\rho_{L} \otimes|\psi\rangle\left\langle\left.\psi\right|_{R C} \text { for } \mid \psi\right\rangle_{R C} \in \mathcal{H}_{R} \otimes \mathcal{H}_{C}$.

In [10], the authors applied Proposition 1.2 to study the saturation of the upper bound of quantum discord. In [11], the authors gave Proposition 1.2 an elementary proof.

In this paper, we study the saturation of the four universal inequalities (1.1) through (1.4).

\section{The saturation of strong subadditivity inequality}

In this section, we give a characterization to the structure of states which saturate the strong subadditivity inequality (1.1) of von Neumann entropy. That is

Theorem 2.1. Let $\sigma_{A B C} \in \mathrm{D}\left(\mathcal{H}_{A} \otimes \mathcal{H}_{B} \otimes \mathcal{H}_{C}\right)$. Then

$$
\mathrm{S}\left(\sigma_{A}\right)+\mathrm{S}\left(\sigma_{C}\right)=\mathrm{S}\left(\sigma_{A B}\right)+\mathrm{S}\left(\sigma_{C B}\right)
$$

if and only if there are two decompositions of system $A$ and $C$, respectively, as

$$
\mathcal{H}_{A}=\bigoplus_{i=1}^{K_{A}} \mathcal{H}_{a_{i}^{L}} \otimes \mathcal{H}_{a_{i}^{R}} \quad \text { and } \quad \mathcal{H}_{C}=\bigoplus_{j=1}^{K_{C}} \mathcal{H}_{c_{j}^{L}} \otimes \mathcal{H}_{c_{j}^{R}}
$$

such that

$$
\sigma_{A B C}=\bigoplus_{i, j} \mu_{i j} \sigma_{a_{i}^{L} B c_{j}^{L}} \otimes \sigma_{a_{i}^{R} c_{j}^{R}}
$$

where $\sigma_{a_{i}^{L} B c_{j}^{L}} \equiv|\psi\rangle\left\langle\left.\psi\right|_{a_{i}^{L} B c_{j}^{L}} \in \mathrm{D}\left(\mathcal{H}_{a_{i}^{L}} \otimes \mathcal{H}_{B} \otimes \mathcal{H}_{c_{j}^{L}}\right), \sigma_{a_{i}^{R} c_{j}^{R}} \in \mathrm{D}\left(\mathcal{H}_{a_{i}^{R}} \otimes \mathcal{H}_{c_{j}^{R}}\right)\right.$ and $\left\{\mu_{i j}\right\}$ is a joint probability distribution.

Proof. We introduce a reference system $D$ such that $\sigma_{A B C D}$ is a purification of $\sigma_{A B C}$. Thus Equation (2.1) can be rewritten into

$$
\mathrm{S}\left(\sigma_{A}\right)+\mathrm{S}\left(\sigma_{C}\right)=\mathrm{S}\left(\sigma_{C D}\right)+\mathrm{S}\left(\sigma_{A D}\right)
$$

It can be seen that, when the systems $A$ and $C$ are fixed, the systems $B$ and $D$ play a symmetric role in Equation (2.1) and Equation (2.4). Analogously, we have

$$
\begin{aligned}
& \mathrm{S}\left(\sigma_{A}\right)+\mathrm{S}\left(\sigma_{A B D}\right)=\mathrm{S}\left(\sigma_{A B}\right)+\mathrm{S}\left(\sigma_{A D}\right), \\
& \mathrm{S}\left(\sigma_{C B D}\right)+\mathrm{S}\left(\sigma_{C}\right)=\mathrm{S}\left(\sigma_{C D}\right)+\mathrm{S}\left(\sigma_{C B}\right) .
\end{aligned}
$$


Again, when the systems $B$ and $D$ are fixed, the systems $A$ and $C$ play a symmetric role in Equation (2.5) and Equation (2.6).

Now it follows from Proposition 1.1 that there are two decompositions of $A$ and $C$, respectively,

$$
\mathcal{H}_{A}=\bigoplus_{i=1}^{K_{A}} \mathcal{H}_{a_{i}^{L}} \otimes \mathcal{H}_{a_{i}^{R}} \quad \text { and } \quad \mathcal{H}_{C}=\bigoplus_{j=1}^{K_{C}} \mathcal{H}_{c_{j}^{L}} \otimes \mathcal{H}_{c_{j}^{R}}
$$

such that

$$
\sigma_{A B D}=\bigoplus_{i} p_{i} \sigma_{a_{i}^{L} B} \otimes \sigma_{a_{i}^{R} D} \quad \text { and } \quad \sigma_{B C D}=\bigoplus_{j} q_{j} \sigma_{B c_{j}^{L}} \otimes \sigma_{c_{j}^{R} D}
$$

Thus $\sigma_{A B C}$ must be of the form:

$$
\sigma_{A B C}=\bigoplus_{i, j} \mu_{i j} \sigma_{a_{i}^{L} B c_{j}^{L}}^{(i j)} \otimes \sigma_{a_{i}^{R} c_{j}^{R}}^{(i j)}
$$

where

$$
\mathrm{S}\left(\sigma_{a_{i}^{L} B}^{(i j)}\right)+\mathrm{S}\left(\sigma_{B c_{j}^{L}}^{(i j)}\right)=\mathrm{S}\left(\sigma_{a_{i}^{L}}^{(i j)}\right)+\mathrm{S}\left(\sigma_{c_{j}^{L}}^{(i j)}\right) \quad(\forall i, j)
$$

Without loss of generality, we assume that the system $a_{i}^{L}$ and $c_{j}^{L}$ can not decomposed like the $\mathcal{H}_{A}$ and $\mathcal{H}_{C}$, respectively. Therefore $\sigma_{a_{i}^{L} B c_{j}^{L}}$ must be a pure state, which implies that

$$
\sigma_{a_{i}^{L} B c_{j}^{L}} \equiv|\psi\rangle\left\langle\left.\psi\right|_{a_{i}^{L} B c_{j}^{L}}\right.
$$

Conversely, if the state $\sigma_{A B C}$ has the form of Equation (2.3), then it is easy to check that Equation (2.1) holds.

We would like to point out that if $\sigma_{A B C}$ is a pure state, by Proposition 1.1, then there must exist a decomposition of $\sigma_{A B C}$ such that its substates are locally pure states.

\section{The saturation of coherent information inequality}

In this section, we make an attempt towards the saturation of coherent information inequality (1.2).

Theorem 3.1. Let $\rho \in \mathrm{D}(\mathcal{H})$ and $\Phi$ be a quantum channel defined over $\mathcal{H}$. Then $I_{c}(\rho, \Phi)=\mathrm{S}(\rho)$ if and only if the following statements hold:

(i) The Hilbert space $\mathcal{H}$ can be decomposed into $\mathcal{H}=\mathcal{H}_{L} \otimes \mathcal{H}_{R}$; 
(ii) The output state $\Phi(\rho)$ of the quantum channel $\Phi$ is a product state: $\Phi(\rho)=\rho_{L} \otimes \rho_{R}$, where $\rho_{L} \in \mathrm{D}\left(\mathcal{H}_{L}\right), \rho_{R} \in \mathrm{D}\left(\mathcal{H}_{R}\right)$.

Proof. Note that

$$
\mathrm{S}(\widehat{\Phi}(\rho))=\mathrm{S}\left(\left(\mathbb{1}_{A} \otimes \Phi\right)\left(\left|\mathbf{u}_{\rho}\right\rangle\left\langle\mathbf{u}_{\rho}\right|\right)\right)
$$

where $\left|\mathbf{u}_{\rho}\right\rangle$ is a purification of $\rho$ in a larger Hilbert space $\mathcal{H}_{A} \otimes \mathcal{H}_{B}$, where $\mathcal{H}_{B} \equiv \mathcal{H}$. It was shown that there exists a quantum channel $\Psi$ (see [8]) such that

$$
I_{c}(\rho, \Phi)=S(\rho) \Longleftrightarrow\left(\mathbb{1}_{A} \otimes \Psi \circ \Phi\right)\left(\left|\mathbf{u}_{\rho}\right\rangle\left\langle\mathbf{u}_{\rho}\right|\right)=\left|\mathbf{u}_{\rho}\right\rangle\left\langle\mathbf{u}_{\rho}\right| .
$$

By the Stinespring dilation theorem (see [5]), we may assume that

$$
\Phi(\rho)=\operatorname{Tr}_{C}\left(U(\rho \otimes|0\rangle\langle 0|) U^{+}\right), \quad U \in \mathrm{U}\left(\mathcal{H}_{B} \otimes \mathcal{H}_{C}\right),|0\rangle \in \mathcal{H}_{C},
$$

which indicates that

$$
\begin{aligned}
\mathbb{1}_{A} \otimes \Phi\left(\left|\mathbf{u}_{\rho}\right\rangle\left\langle\mathbf{u}_{\rho}\right|\right) & =\operatorname{Tr}_{C}\left(\left(\mathbb{1}_{A} \otimes U\right)\left(\left|\mathbf{u}_{\rho}\right\rangle\left\langle\mathbf{u}_{\rho}|\otimes| 0\right\rangle\langle 0|\right)\left(\mathbb{1}_{A} \otimes U\right)^{\dagger}\right) \\
& =\operatorname{Tr}_{C}(|\Omega\rangle\langle\Omega|)
\end{aligned}
$$

where $|\Omega\rangle \stackrel{\text { def }}{=}\left(\mathbb{1}_{A} \otimes U\right)\left(\left|\mathbf{u}_{\rho}\right\rangle \otimes|0\rangle\right)$. Now

$$
|\Omega\rangle\langle\Omega|=\left(\mathbb{1}_{A} \otimes U\right)\left(\left|\mathbf{u}_{\rho}\right\rangle\left\langle\mathbf{u}_{\rho}|\otimes| 0\right\rangle\langle 0|\right)\left(\mathbb{1}_{A} \otimes U\right)^{\dagger}
$$

is a tripartite state on $\mathcal{H}_{A} \otimes \mathcal{H}_{B} \otimes \mathcal{H}_{C}$, it follows that

$$
\begin{aligned}
\operatorname{Tr}_{C}(|\Omega\rangle\langle\Omega|) & =\mathbb{1}_{A} \otimes \Phi\left(\left|\mathbf{u}_{\rho}\right\rangle\left\langle\mathbf{u}_{\rho}\right|\right) \equiv \Omega_{A B} \\
\operatorname{Tr}_{A}(|\Omega\rangle\langle\Omega|) & =U(\rho \otimes|0\rangle\langle 0|) U^{+} \equiv \Omega_{B C} \\
\operatorname{Tr}_{A C}(|\Omega\rangle\langle\Omega|) & =\Phi(\rho) \equiv \Omega_{B}
\end{aligned}
$$

where $\Omega_{A B C} \equiv|\Omega\rangle\langle\Omega|$. From the above expressions, it is obtained that

$$
\begin{aligned}
\mathrm{S}\left(\Omega_{A B C}\right) & =0 \\
\mathrm{~S}\left(\Omega_{B}\right) & =\mathrm{S}(\Phi(\rho)) \\
\mathrm{S}\left(\Omega_{B C}\right) & =\mathrm{S}(\rho), \\
\mathrm{S}\left(\Omega_{A B}\right) & =\mathrm{S}\left(\left(\mathbb{1}_{A} \otimes \Phi\right)\left(\left|\mathbf{u}_{\rho}\right\rangle\left\langle\mathbf{u}_{\rho}\right|\right)\right)
\end{aligned}
$$

Apparently, $I_{c}(\rho, \Phi)=\mathrm{S}(\rho) \Longleftrightarrow \mathrm{S}(\Phi(\rho))=\mathrm{S}\left(\left(\mathbb{1}_{A} \otimes \Phi\right)\left(\left|\mathbf{u}_{\rho}\right\rangle\left\langle\mathbf{u}_{\rho}\right|\right)\right)+\mathrm{S}(\rho)$, that is,

$$
\begin{aligned}
I_{\mathcal{c}}(\rho, \Phi)=\mathrm{S}(\rho) & \Longleftrightarrow \mathrm{S}\left(\Omega_{B}\right)=\mathrm{S}\left(\Omega_{A B}\right)+\mathrm{S}\left(\Omega_{B C}\right) \\
& \Longleftrightarrow \mathrm{S}\left(\Omega_{B}\right)-\mathrm{S}\left(\Omega_{C}\right)=\mathrm{S}\left(\Omega_{B C}\right) .
\end{aligned}
$$

It follows from Proposition 1.2 that this equality holds if and only if 
(i) $\mathcal{H}_{B}$ can be factorized into the form $\mathcal{H}_{B}=\mathcal{H}_{L} \otimes \mathcal{H}_{R}$,

(ii) $\Omega_{B C}=\rho_{L} \otimes|\psi\rangle\left\langle\left.\psi\right|_{R C} \text { for } \mid \psi\right\rangle_{R C} \in \mathcal{H}_{R} \otimes \mathcal{H}_{C}$.

That is,

$$
\Phi(\rho)=\operatorname{Tr}_{C}\left(\rho_{L} \otimes|\psi\rangle\left\langle\left.\psi\right|_{R C}\right)=\rho_{L} \otimes \rho_{R}\right.
$$

This indicates that if the coherent information arrives at $S(\rho)$, then the output state $\Phi(\rho)$ of the quantum channel $\Phi$ is a product state.

\section{The saturation of Holevo quantity and average entropy inequalities}

In this section, we study the saturation of Holevo quantity inequality (1.3) and average entropy inequality (1.4) which are induced by a quantum channel.

Theorem 4.1. With the above notation, we have the following result:

$$
\chi\left(\left\{q_{\mu}, \rho_{\mu}^{\prime}\right\}\right)=\mathrm{S}(\widehat{\Phi}(\rho)) \text { if and only if } \Phi(\rho)=\bigoplus_{i, j} p_{i j} \omega_{a_{i}^{L}}^{(i j)} \otimes \omega_{a_{i}^{R}}^{(i j)}
$$

Proof. In order to prove the conclusion, we need to go back to the original proof of [2]. Where the authors introduced a tripartite state

$$
\omega_{A B C} \stackrel{\text { def }}{=} \sum_{\mu, v}\left(K_{\mu} \rho K_{v}^{+}\right)_{A} \otimes|\mu\rangle\left\langle\left.\nu\right|_{B} \otimes \mid \mu\right\rangle\left\langle\left. v\right|_{C} .\right.
$$

From the above expression, we see that $\omega_{A B C}$ is a symmetric state for $B C$ relative to $A$ and $\mathcal{H}_{B}=\mathcal{H}_{C}$. Denote $q_{\mu}=\operatorname{Tr}\left(K_{\mu} \rho K_{\mu}^{+}\right)$and $\rho_{\mu}^{\prime}=q_{\mu}^{-1} K_{\mu} \rho K_{\mu}^{+}$. Since

$$
\mathrm{S}\left(\omega_{B C}\right)=\mathrm{S}(\widehat{\Phi}(\rho)), \quad \mathrm{S}\left(\omega_{A}\right)=\mathrm{S}\left(\sum_{\mu} q_{\mu} \rho_{\mu}^{\prime}\right), \quad \sum_{\mu} q_{\mu} \mathrm{S}\left(\rho_{\mu}^{\prime}\right)=\mathrm{S}\left(\omega_{A C}\right)-\mathrm{S}\left(\omega_{B}\right) .
$$

Therefore $\chi\left(\left\{q_{\mu}, \rho_{\mu}^{\prime}\right\}\right)=\mathrm{S}(\widehat{\Phi}(\rho))$ if and only if $\mathrm{S}\left(\omega_{A}\right)+\mathrm{S}\left(\omega_{C}\right)=\mathrm{S}\left(\omega_{A B}\right)+\mathrm{S}\left(\omega_{B C}\right)$. This amounts to say, by Theorem 2.1 , that

$$
\omega_{A B C}=\sum_{\mu, v}\left(K_{\mu} \rho K_{v}^{\dagger}\right)_{A} \otimes|\mu\rangle\left\langle\left. v\right|_{B} \otimes \mid \mu\right\rangle\left\langle\left. v\right|_{C}=\bigoplus_{i, j} p_{i j} \omega_{a_{i}^{L} B c_{j}^{L}}^{(i j)} \otimes \omega_{a_{i}^{R} c_{j}^{R}}^{(i j)}\right.
$$

where each $\omega_{a_{i}^{L} B c_{j}^{L}}^{(i j)}$ is a pure state. Since both $B$ and $C$ are identical, it follows that

$$
\sum_{\mu} K_{\mu} \rho K_{\mu}^{\dagger} \otimes|\mu\rangle\langle\mu|=\bigoplus_{i, j} p_{i j} \omega_{a_{i}^{L} c_{j}^{L}}^{(i j)} \otimes \omega_{a_{i}^{R} c_{j}^{R}}^{(i j)}
$$


which implies that

$$
\Phi(\rho)=\omega_{A}=\bigoplus_{i, j} p_{i j} \omega_{a_{i}^{L}}^{(i j)} \otimes \omega_{a_{i}^{R}}^{(i j)}
$$

From this expression, it is seen that the output state of a quantum channel $\Phi$ with input state $\rho$ is a weighted state of block diagonal form in some basis.

Theorem 4.2. The average entropy attains the entropy of the initial state $\rho$, that is, $\sum_{\mu} q_{\mu} S\left(\rho_{\mu}^{\prime}\right)=S(\rho)$ if and only if $\Phi(*)=U(*) U^{\dagger}$ for some unitary operator $U$.

Proof. Note that $\sum_{\mu} q_{\mu} \mathrm{S}\left(\rho_{\mu}^{\prime}\right)=\mathrm{S}(\rho)$ if and only if $\mathrm{S}\left(\omega_{A C}\right)-\mathrm{S}\left(\omega_{B}\right)=\mathrm{S}\left(\omega_{A B C}\right)=\mathrm{S}\left(\omega_{A B}\right)-\mathrm{S}\left(\omega_{C}\right)$. This amounts to say, by Proposition 1.2, that

$$
\omega_{A B C}=\omega_{L} \otimes|\psi\rangle\left\langle\left.\psi\right|_{R C}, \quad \omega_{A C B}=\omega_{\hat{L}} \otimes \mid \psi\right\rangle\left\langle\left.\psi\right|_{\hat{R} B} .\right.
$$

Since $\omega_{A B C}=\omega_{A C B}$, in fact, the composite system of $B$ and $C$ stays in a symmetric state when we ignore system $A$, that is, swapping the role of $B$ and $C$ leaves $\omega_{A B C}$ invariant, it follows that

$$
\omega_{A}=\omega_{L}=\omega_{\hat{L}^{\prime}} \quad \omega_{R}=\omega_{B}, \quad \omega_{C}=\omega_{\hat{R}}
$$

This fact indicates that system $A$ has no correlation with the composite system of $B$ and $C$, which is in a pure symmetric state $|\psi\rangle\left\langle\left.\psi\right|_{B C}\right.$ on $\mathcal{H}_{B} \otimes \mathcal{H}_{C}$ with $\mathcal{H}_{B}=\mathcal{H}_{C}$. Thus, we have $\omega_{A B C}=$ $\omega_{A} \otimes \omega_{B C}$ with $\omega_{B C}=|\psi\rangle\left\langle\left.\psi\right|_{B C}\right.$, that is,

$$
|\psi\rangle\left\langle\left.\psi\right|_{B C}=\sum_{\mu, v} \operatorname{Tr}\left(K_{\mu} \rho K_{v}^{+}\right) \mid \mu\right\rangle\langle v|\otimes| \mu\rangle\langle\nu| .
$$

Now $W|\mu\rangle=|\mu \mu\rangle$ for all $\mu$ defines an isometry $W$, and

$$
|\psi\rangle\left\langle\left.\psi\right|_{B C}=W\left(\sum_{\mu, \nu} \operatorname{Tr}\left(K_{\mu} \rho K_{v}^{\dagger}\right)|\mu\rangle\langle v|\right) W^{\dagger} .\right.
$$

Therefore, the von Neumann entropy of $\sum_{\mu, v} \operatorname{Tr}\left(K_{\mu} \rho K_{v}^{\dagger}\right)|\mu\rangle\langle v|$ is vanished since isometric transformation leaves the von Neumann entropy of state invariant. This indicates that

$$
\sum_{\mu, v} \operatorname{Tr}\left(K_{\mu} \rho K_{v}^{+}\right)|\mu\rangle\langle\nu| \equiv \widehat{\Phi}(\rho)
$$

is still a pure state. Define an isometry $V$ as follows:

$$
V|\phi\rangle=K_{\mu}|\phi\rangle \otimes|\mu\rangle, \quad \forall \mu .
$$

It follows that

$$
V \rho V^{\dagger}=\sum_{\mu, v} K_{\mu} \rho K_{v}^{\dagger} \otimes|\mu\rangle\langle v|
$$


implying that $\Phi(\rho)=\operatorname{Tr}_{2}\left(V \rho V^{\dagger}\right)$ and $\widehat{\Phi}(\rho)=\operatorname{Tr}_{1}\left(V \rho V^{\dagger}\right)$. Note that $\widehat{\Phi}(\rho)$ is a pure state, the bipartite state $\sum_{\mu, v} K_{\mu} \rho K_{v}^{\dagger} \otimes|\mu\rangle\langle v|$ is a product state: $V \rho V^{\dagger}=\operatorname{Tr}_{2}\left(V \rho V^{\dagger}\right) \otimes \operatorname{Tr}_{1}\left(V \rho V^{\dagger}\right)$. Therefore

$$
\sum_{\mu, v} K_{\mu} \rho K_{v}^{\dagger} \otimes|\mu\rangle\langle v|=\Phi(\rho) \otimes \widehat{\Phi}(\rho) .
$$

Again since $\widehat{\Phi}(\rho)$ is a pure state, there must exist complex numbers $\lambda_{\mu}$ such that $\operatorname{Tr}\left(K_{\mu} \rho K_{v}^{\dagger}\right)=$ $\lambda_{\mu} \bar{\lambda}_{\nu}$ for complex numbers $\lambda_{\mu}$. Clearly $\sum_{\mu}\left|\lambda_{\mu}\right|^{2}=1$. Now we can infer from Equation (4.4) that

$$
\Phi(\rho)=\left(\lambda_{\mu}^{-1} K_{\mu}\right) \rho\left(\lambda_{v}^{-1} K_{v}\right)^{\dagger}=\left(\lambda_{v}^{-1} K_{v}\right) \rho\left(\lambda_{\mu}^{-1} K_{\mu}\right)^{\dagger}, \quad \forall \mu, v
$$

or

$$
K_{\mu} \rho K_{v}^{\dagger}=\lambda_{\mu} \bar{\lambda}_{v} \Phi(\rho),
$$

which implies that

$$
\begin{aligned}
\rho & =\left(\sum_{\mu} K_{\mu}^{\dagger} K_{\mu}\right) \rho\left(\sum_{v} K_{v}^{\dagger} K_{v}\right)=\sum_{\mu, v} K_{\mu}^{\dagger}\left(K_{\mu} \rho K_{v}^{\dagger}\right) K_{v} \\
& =\left(\sum_{\mu} \lambda_{\mu} K_{\mu}^{\dagger}\right) \Phi(\rho)\left(\sum_{v} \lambda_{v} K_{v}^{\dagger}\right)^{\dagger} \equiv M \Phi(\rho) M^{\dagger}
\end{aligned}
$$

where $M \stackrel{\text { def }}{=} \sum_{\mu} \lambda_{\mu} K_{\mu}^{+}$. From Equation (4.4), we can see that

$$
\mathrm{S}(\rho)=\mathrm{S}\left(V \rho V^{\dagger}\right)=\mathrm{S}(\Phi(\rho))+\mathrm{S}(\widehat{\Phi}(\rho))=\mathrm{S}(\Phi(\rho))
$$

since $\widehat{\Phi}(\rho)$ is a pure state. Moreover, we can have $I_{c}(\rho, \Phi)=S(\rho)$. This showed that

$$
\Phi(\rho)=\rho_{L} \otimes \rho_{R}
$$

In the above process, the output state of the complementary channel is pure state. Without loss of generality, we assume that the environment starts in a pure state, this implies that the complementary channel is an unitary channel. From the basic properties of quantum channel, we obtain that $\sum_{\mu} q_{\mu} \mathrm{S}\left(\rho_{\mu}^{\prime}\right)=\mathrm{S}(\rho)$ if and only if the quantum channel $\Phi$ is the unitary channel.

\section{Concluding remarks}

In this paper we give characterizations of several famous quantum information inequalities when becoming equalities. Specifically, we characterize the saturation of four universal inequalities in quantum information theory, including a variant version of strong subadditivity inequality for von Neumann entropy, the coherent information inequality, the Holevo quantity and average entropy inequalities. The proofs are based on the works of Hayden etc. [8], and that of Zhang and $\mathrm{Wu}[9]$. These results shed new light on quantum information inequalities. 
In the future research, we will investigate the approximate version of the above-discussed information inequalities since the approximate information inequalities are more or less related to entanglement theory.

Acknowledgement. We want to express our heartfelt thanks to Wojciech Roga for useful comments. This work is supported by National Natural Science Foundation of China (11301124, 11171301) and the Doctoral Programs Foundation of Ministry of Education of China (J20130061).

\section{References}

[1] E.H. Lieb and M.B. Ruskai. Proof of the strong subadditivity of quantum-mechanical entropy. J. Math. Phys. 14, 1938-1941 (1973).

[2] W. Roga, M. Fannes, and K. Życzkowski. Universal bounds for the Holevo quantity, coherent information, and the Jensen-Shannon divergence. Phys. Rev. Lett. 105, 040505 (2010).

[3] G. Lindblda. Completely positive maps and entropy inequalities. Commun. Math. Phys. 40, 147-151 (1975).

[4] M. Nielsen and I. Chuang. Quantum Computation and Quantum Information. Cambridge Series on Information and the Natural Sciences. Cambridge University Press, 2000.

[5] J. Watrous, Theory of Quantum Information, University of Waterloo, Waterloo (2008). See http://www.cs.uwaterloo.ca/ watrous/quant-info/

[6] T.S. Cubitt, M.B. Ruskai, G. Smith, The structure of degradable quantum channels, J. Math. Phys. 49, 102104 (2008).

[7] A.S. Holevo, V. Giovannetti, Quantum channels and their entropic characteristics, Rep. Prog. Phys. 75, 046001 (2012).

[8] P. Hayden, R. Jozsa, D. Petz and A. Winter. Structure of states which satisfy strong subadditivity of quantum entropy with equality. Commun. Math. Phys. 246, 359-374 (2004).

[9] L. Zhang and J. Wu. On Conjectures of Classical and Quantum Correlations in Bipartite States. J. Phys. A: Math. Theor. 45, 025301(2012).

[10] Z. Xi, X. Lu, X. Wang, and Y. Li. Necessary and sufficient condition for saturating the upper bound of quantum discord. Phys. Rev. A 85, 032109 (2012).

[11] E.A. Carlen and E.H. Lieb. Bounds for entanglement via an extension of strong subadditivity of entropy. Lett. Math. Phys. 101, 1-11 (2012). 